\title{
Estudo comparativo de ferramentas colaborativas online para facilitação visual
}

\author{
Comparative study of online collaborative tools for visual facilitation
}

Reinaldo Pereira de Moraes, Claudia Mont'Alvão, Stephania Padovani

critérios ergonômicos, representação gráfica, construção colaborativa de conhecimento, pensamento visual, colaboração remota

Este artigo aborda, sob a ótica do Design da Informação, a realização de dinâmicas colaborativas de discussão visual auxiliadas por ferramentas online. Nesse contexto, o objetivo deste trabalho é comparar as ferramentas online para facilitação visual Jamboard, Miro e Mural baseado nas Heurísticas de Usabilidade de Nielsen e elencar as suas principais características e recursos colaborativos necessários para a facilitação remota de dinâmicas colaborativas. Para tanto, foram adotados como procedimentos metodológicos um pré-diagnóstico baseado nas heurísticas de usabilidade, dinâmicas colaborativas e entrevista semiestruturada com 3 participantes. O pré-diagnóstico foi realizado pelo pesquisador e revisado ao observar o desenvolvimento das dinâmicas. Os participantes foram organizados em três duplas a fim de realizar as dinâmicas usando as ferramentas selecionadas. As sessões tiveram duração de 90 minutos. Na primeira metade de cada sessão, a dupla elaborou as três representações gráficas solicitadas pelo pesquisador. Na segunda metade, a dupla foi entrevistada sobre o uso da ferramenta, funções disponibilizadas e possíveis melhorias em termos de colaboração. Como resultados, foi possível identificar os recursos necessários para a condução de dinâmicas colaborativas online e sugerir a incorporação de recursos que não foram disponibilizados ou encontrados nas ferramentas durante as dinâmicas colaborativas.

ergonomic criteria, graphic representation, collaborative knowledge construction, visual thinking, remote collaboration

From the perspective of Information Design, this paper addresses the realization of collaborative dynamics of visual discussion aided by online tools. In this context, this work aims to compare the online tools for visual facilitation Jamboard, Miro, and Mural based on Nielsen's Usability Heuristics and to list their main collaborative features and needed resources to facilitate collaborative dynamics online. A pre-diagnosis based on heuristic usability, collaborative dynamics, and a semi-structured interview with 3 participants were adopted as methodological procedures. The pre-diagnosis heuristic was performed by the researcher and reviewed when observing the development of dynamics. The participants were organized into three pairs in order to perform the dynamics using the selected tools. Sessions lasted 90 minutes. In the first half of each session, the duo prepared the three graphic representations requested by the researcher. In the second half, the duo was interviewed about the tool usage, available functions, and possible improvements in terms of collaboration. As a result, it was possible to identify the resources needed to conduct collaborative dynamics online and suggest incorporating resources that were not made available or found in the tools during the collaborative dynamics.

Anais do $10^{\circ} \mathrm{CIDI}$ e $10^{\circ} \mathrm{CONGIC}$

Kelli C.A.S. Smythe, Rafael de Castro Andrade (orgs.)

Sociedade Brasileira de Design da Informação - SBDI

Curitiba | Brasil | 2021
Proceedings of the $10^{\text {th }} \mathrm{CIDI}$ and $10^{\text {th }}$ CONGIC

Kelli C.A.S. Smythe, Rafael de Castro Andrade (orgs.)

Sociedade Brasileira de Design da Informação - SBDI Curitiba | Brazil | 2021 


\section{Introdução}

Embora o trabalho colaborativo remoto não seja necessariamente consequência da pandemia provocada pela COVID-19, não se pode ignorar o fato de que essa modalidade de trabalho, outrora latente, tem sido colocada em evidência e levado as instituições a se adaptarem a este contexto conforme suas próprias (e diferentes) demandas de colaboração. Os cenários podem ser os mais diversos, tais como: (a) um grupo de 3 ou 4 pessoas trabalhando simultaneamente no mesmo documento; (b) pessoas trabalhando individualmente em seus arquivos e acompanhando o desenvolvimento do trabalho uns dos outros; (c) pessoas que necessitam se comunicar e manter um projeto atualizado.

As ferramentas utilizadas na execução do trabalho são tão diversas quanto os cenários colaborativos exemplificados anteriormente. A Web 2.0 trouxe consigo a possibilidade de transpor os chamados softwares desktop para o ambiente online. Com isso, um texto, que para ser finalizado colaborativamente deveria ser escrito por uma pessoa, revisado por outra e formatado por uma terceira - cada um trabalhando individualmente em seu equipamento agora pode ser escrito, revisado e formatado enquanto as pessoas trabalham simultaneamente no documento. A alteração da dinâmica do trabalho colaborativo desse exemplo também pode ser observada em outros tipos de documentos e contextos, desde planilhas eletrônicas, bancos de dados, elaboração de slides e manipulação de imagens para diversos fins até a realização de reuniões online por meio de videoconferências acompanhadas (ou não) de compartilhamento de telas e/ou ferramentas que permitam a colaboração síncrona ou assíncrona. Diante desta variedade de contextos, aponta-se o uso de ferramentas online facilitadoras da discussão visual como um recorte estudado neste artigo. Nesse ambiente virtual, as pessoas podem usar diferentes ferramentas de software, tais como Jamboard, Miro e Mural, capazes de promover a facilitação visual a fim de expressar suas ideias com o auxílio de recursos representacionais pré-definidos que permitem a elaboração de representações gráficas.

Este artigo tem como objetivos realizar um pré-diagnóstico de três ferramentas online para facilitação visual com base nas Heurísticas de Usabilidade de Nielsen (1990), identificar suas principais características e apontar quais recursos colaborativos são necessários para a facilitação de dinâmicas colaborativas de discussão visual.

\section{Contexto teórico}

\section{Pensamento visual e construção colaborativa do conhecimento}

A Construção Colaborativa de Conhecimento pode ser entendida como um processo de cunho comunicacional e social com a intenção de co-criar artefatos de conhecimento (Stahl, 2006) ou como um processo de construção ativa de significados por meio do desenvolvimento de objetos compartilhados (Singh et al., 2009). Embora estes autores tenham expressado seus conceitos de Construção Colaborativa de Conhecimento de formas diferentes, um como um 
processo comunicacional com a intenção de co-criar e o outro como um processo de construção mediado pelo compartilhamento de objetos, em ambos os conceitos pode-se entender que na Construção Colaborativa de Conhecimento o processo coletivo de criação e geração de novos conhecimentos está em constante transformação apoiada pela interação ativa entre os participantes e mediada por artefatos.

Já o pensamento visual (visual thinking) pode ser definido como a habilidade de usar imagens e gráficos para esclarecer e representar pensamentos, ideias e dados (Cyrs, 1997) ou a habilidade de descobrir ideias invisíveis e compartilhá-las por meio de imagens com outras pessoas para que elas as compreendam (Roam, 2009). Embora nem todas as pessoas sejam treinadas na arte da representação por meio de imagens, Gray (2012) destaca que qualquer pessoa tem esta capacidade de expressar pensamentos através de representações gráficas mesmo não sendo excelentes desenhistas. Este pensamento é corroborado por Arnheim (1969), para quem a percepção de formas marca o começo da formação de um conceito.

Aliando-se os dois conceitos (pensamento visual e Construção Colaborativa de Conhecimento), a experiência visual pode ser compreendida como um processo de construção de um conhecimento específico no qual toda forma de pensamento (por exemplo, abstrações, significados, conteúdos, conceitos e atividades) é passível de ser expressada visualmente por meio de representações gráficas. Entende-se representação gráfica como um artefato visível registrado em superfície mais ou menos plana e elaborado a fim de expressar algum tipo de informação (Engelhardt, 2002).

Apoiados na ideia de Construção Colaborativa de Conhecimento como um recurso comunicacional (Stahl, 2006) por meio do desenvolvimento de objetos compartilhados (Singh et al., 2009), neste artigo, denomina-se dinâmicas colaborativas de discussão visual aqueles momentos nos quais dois ou mais indivíduos estão presentes em um ambiente físico ou virtual interagindo ativamente de forma síncrona no exercício do pensamento visual.

\section{Facilitação gráfica}

A facilitação gráfica pode ser observada historicamente a partir das mudanças ocorridas em termos de influências de recursos visuais no desenvolvimento organizacional, de publicações inovadoras e de novas tecnologias impactantes, dividido em dois espaços temporais destacáveis: da década de 1940 à década de 1970 e da década de 1980 aos anos 2000 (Valenza \& Adkins, 2009). No primeiro espaço temporal, esses autores apontam diversos pesquisadores e trabalhos que estariam voltados para o lado humano dos negócios. Quanto aos materiais e equipamentos utilizados na facilitação gráfica, o flip chart se manteve como único recurso até a década de 1960 quando surgiram os murais de papel. Em seguida, na década de 1970 surgiram o post-it e o quadro branco. O segundo espaço temporal se inicia na década de 1980. A esse período os autores associam diversos pesquisadores e trabalhos indicando que as ferramentas de pensamento visual para negócios estão se tornando populares. Quanto aos materiais e equipamentos utilizados na facilitação gráfica, devido ao início do uso de computadores pessoais (década de 1980), ao advento da internet (década de 1990), e ao surgimento dos laptops e tablets (década 2000), foi possível agregar à essas 
transformações tecnológicas o conhecimento de Design da Informação. A conectividade da internet permitiu uma realidade de acesso instantâneo a uma infinidade de multicanais de dados e informações, tais como reuniões virtuais, redes sociais, mapas clicáveis, sistemas colaborativos de construção de conhecimento, cujas características de uso parecem possibilitar múltiplas visões, constantes reavaliações, e mudança na visão de mundo dos que as utilizam.

Apoiada no pensamento visual e em outras teorias e tecnologias, a facilitação gráfica articula imaginação e metáfora como formas de extração e representação das reflexões sobre os padrões de organização e processos de um grupo, contribuindo para que as pessoas compreendam os seus significados (Sibbet, 2006) por meio da organização e representação do conhecimento (Novak; Cañas, 2006). Nesse sentido, a metacognição está envolvida no processo de facilitação visual na medida em que, segundo Bertolini e Silva (2005, p. 53), "se refere à capacidade do indivíduo pensar sobre os seus próprios pensamentos, levando em conta alcançar níveis mais altos de autoconsciência", possibilitando ao indivíduo "[...] ser capaz de conhecer e de autorregular o seu funcionamento cognitivo com a finalidade de solucionar problemas" (Bertolini \& Silva, 2005, p. 53).

Sendo mais pragmático, Gray (2012) argumenta que se uma ideia não pode ser representada, ele não pode ser executada. Além disso, afirma que a capacidade de elaborar representações gráficas a fim de expor ideias é inerente a qualquer pessoa, sugerindo um conjunto de 12 glifos básicos para que todas as pessoas possam representar pensamentos, apesar de não serem desenhistas habilidosos. Nesse sentido, Gray (2012) organizou uma matriz que exemplifica de forma clara e concisa a utilização dos glifos combinados entre si a fim de representar diversas ideias (Figura 1).

Figura 1: Utilização dos glifos para representar ideias, baseada em Gray (2012).

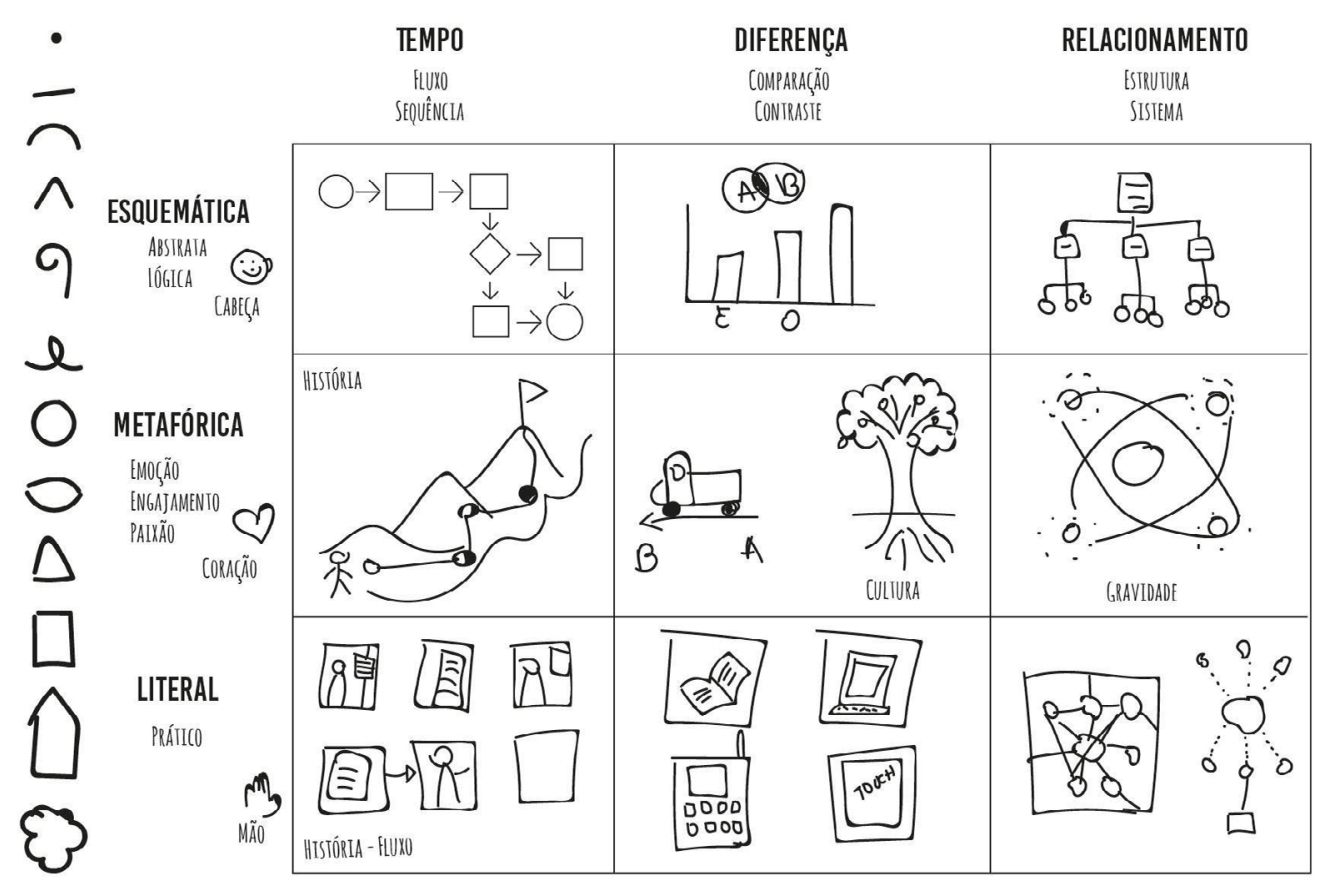




\section{Ferramentas online facilitadoras da discussão visual}

A sugestão de usar glifos básicos para representar ideias também é válida no contexto virtual. Porém, além dos glifos básicos, no contexto virtual também é possível usar outros recursos pré-definidos disponíveis em ferramentas que permitem a elaboração de representações. Neste artigo serão estudadas três softwares, as quais são consideradas como ferramentas online facilitadoras da discussão visual: Jamboard, Miro e Mural.

Jamboard é um quadro branco digital que oferece uma experiência colaborativa para equipes e salas de aula. É possível criar um quadro, editá-lo em seu dispositivo e compartilhálo com outros usuários, possibilitando a colaboração de forma síncrona ou assíncrona (GOOGLE, n.d.).

Miro é uma plataforma colaborativa online para reunir equipes a fim de criar, colaborar e centralizar a comunicação. Possibilita a realização de brainstorming, a representação de processos e a tomada de decisão em um espaço de trabalho digital por meio da colaboração assíncrona e síncrona (MIRO, n.d.).

Mural é um ambiente de trabalho digital para colaboração visual no qual todos podem trabalhar juntos se comunicando visualmente por meio de lembretes, fluxogramas, mapas, desenhos e outros meios. Além disso, também é possível manter o engajamento e produtividade dos colaboradores com recursos de votação, cronômetro e comemoração (MURAL, n.d.).

Comparando as três ferramentas, observa-se que a Jamboard é uma página em branco que, embora não disponibilize modelos pré-definidos de diagramas, está incorporada ao GSuite da Google, permite a inserção de imagens e formas básicas, e disponibiliza o download do resultado em PDF. Miro e Mural são plataformas semelhantes entre si em termos de funcionalidades e recursos disponíveis. Ambas possuem modelos pré-configurados de diagramas e mapas para diversas aplicações. Além disso, possibilitam a exportação do resultado do trabalho elaborado no formato de imagem ou PDF. Entretanto, não possuem integração com outras plataformas de comunicação ou gerenciamento de conteúdos.

Uma busca por assunto realizada no portal Periódicos da Capes, usando os termos Miro, Mural e Jamboard associados a graphic facilitation, collaborative drawing e visual collaboration, identificou três trabalhos de pesquisa. Dois desses trabalhos envolvem o uso da ferramenta Miro e um relata o uso da ferramenta Mural. Nenhum resultado descreve o uso da Jamboard em pesquisas no contexto da facilitação/colaboração visual.

Hardy e Garfen (2020) relatam como conduziram trabalhos de inovação e colaboração remotamente usando a ferramenta Miro para apoiar o desenvolvimento de produtos digitais. Como resultado desta prática, elas acreditam que o compartilhamento de recursos visuais reforça as recomendações e as apoia nas explicações sobre a tomada de decisão. Howe et al. (2021) descrevem uma pesquisa envolvendo profissionais de fatores humanos que trabalham na área de saúde. As respostas da pesquisa foram transcritas, segmentadas em depoimentos e exportadas para Miro, onde foram analisadas tematicamente e agrupadas por quatro PFHs em um processo iterativo. 
Roush e Burmeister (2020) descrevem uma prática educacional relacionada ao entendimento sobre o DNA do COVID-19 a partir do dogma central da biologia molecular na tentativa de melhorar a aprendizagem e o envolvimento do aluno promovendo atividades interativas e colaborativas usando a ferramenta Mural.

\section{Procedimentos metodológicos}

A seleção das ferramentas para esta pesquisa foi motivada pelas observações de seus usos como recursos pedagógicos em atividades acadêmicas nas disciplinas cursadas no Programa de Pós-Graduação em Design da Universidade Federal do Paraná.

Para a realização do pré-diagnóstico das ferramentas, foram adotadas as 10 Heurísticas de Usabilidade de Nielsen (1990): Visibilidade do estado do sistema, Correspondência entre o sistema e o mundo real, Controle e liberdade por parte do usuário, Consistência e padrões/convenções, Reconhecimento preferível à memorização, Flexibilidade e eficiência de uso, Estética e design minimalista, Prevenção de erros, Reconhecimento, correção e recuperação de erros, Ajuda/ suporte e documentação.

Esse pré-diagnóstico foi conduzido pelo pesquisador, e revisado durante a observação da realização das dinâmicas colaborativas e da verbalização dos participantes sobre as ferramentas, suas interfaces e funcionalidades.

Para identificar aspectos e características relacionados às funções disponíveis nas ferramentas Jamboard, Miro e Mural que pudessem contribuir com a realização das dinâmicas colaborativas de discussão visual, foram realizadas dinâmicas colaborativas de 45 minutos e entrevistas semiestruturadas em duplas, com duração de 45 minutos, a partir das seguintes perguntas:

1. Vocês possuem algum conhecimento prévio da ferramenta utilizada?

2. Se sim, como vocês consideram seus conhecimentos prévios sobre a ferramenta?

3. O período de 10 minutos antes da realização da dinâmica foi suficiente para aprender/reconhecer as funções necessárias para a execução da tarefa proposta?

4. A ferramenta é adequada ao nível de homogeneidade da dupla (considere interface, ajuda on-line, comandos)?

5. A ferramenta é adequada para a proximidade/conhecimento existente entre a dupla (considere tarefas síncronas)?

6. A ferramenta é adequada para a organização dos participantes (considere aspectos de anonimato e não anonimato das colaborações em grupos maiores)?

7. A ferramenta disponibiliza canais de comunicação adequados para manter a interação entre os participantes?

8. Quais canais seriam necessários?

9. A ferramenta dá suporte para as contribuições do grupo na construção colaborativa?

10. É possível executar ações diferentes e/ou simultâneas sobre o mesmo objeto? 
11. Quais funções ou recursos da ferramenta podem contribuir para o trabalho colaborativo?

12. Quais funções ou recursos relacionados à colaboração seriam necessários para melhorar a ferramenta?

As dinâmicas colaborativas foram realizadas com o auxílio de três participantes, identificados como P1, P2 e P3. Os participantes selecionados (2 homens e 1 mulher) têm idade entre 22 e 30 anos, são estudantes universitários de curso presencial que assistem às aulas remotamente devido à pandemia COVID-19. A pesquisa foi realizada com uma amostra não probabilística de participantes devido à falta de acesso à totalidade de usuários de ferramentas colaborativas online para discussão visual, impossibilitando uma seleção completamente aleatória na qual todos na população teriam as mesmas chances de serem selecionados. Ainda, esta seleção por conveniência foi realizada a partir do convite por email aos contatos da lista de emails do pesquisador. Apesar de a amostra ser considerada não probabilística, dos 43 contatos que se encaixavam no perfil definido anteriormente, 20 contatos foram selecionados randomicamente. $O$ convite foi enviado e apenas 3 responderam confirmando a disponibilidade de participação na pesquisa. Por isso, os três participantes foram organizados em duplas: P1-P2, P2-P3 e P1-P3.

Na realização das dinâmicas, após a explicação da matriz de Gray (2012), as duplas foram orientadas a elaborar representações baseadas na combinação dos tipos de representação (esquemática, metafórica e literal) com as temáticas propostas (tempo, diferença e relacionamento). A dupla P1-P2 utilizou a ferramenta Miro, a dupla P2-P3 utilizou a ferramenta Mural e a dupla P3-P1 utilizou a ferramenta Jamboard.

Foram realizadas três sessões de 90 minutos (45 minutos para a dinâmica colaborativa e 45 minutos para entrevista), agendadas conforme a disponibilidade dos participantes. Para cada sessão, foram fornecidos aos participantes um link de acesso a uma sala de videoconferência da plataforma Zoom e um link para arquivos da ferramenta utilizada, contendo uma versão simplificada da matriz de Gray com os exemplos de representações gráficas que deveriam ser elaboradas (Figura 2, Figura 3 e Figura 4).

Considerando que o objetivo deste estudo não é a avaliação do processo de $\mathrm{C}^{3} \mathrm{em} \mathrm{si}$, mas a identificação de características e recursos colaborativos necessários para a facilitação remota de dinâmicas colaborativas de discussão visual, as duplas puderam escolher entre elaborar a representação de um assunto novo ou reproduzir a representação sugerida na matriz.

Durante as dinâmicas colaborativas, cujas sessões de videoconferência na plataforma Zoom foram gravadas, os participantes foram incentivados a verbalizar suas ações enquanto realizavam a atividade para que fosse possível identificar as demandas dos usuários em termos de colaboração na utilização das ferramentas. Ao final das dinâmicas os participantes foram submetidos à entrevista semiestruturada. 
Figura 2: Representações para serem elaboradas na ferramenta Miro, baseada em Gray (2012).

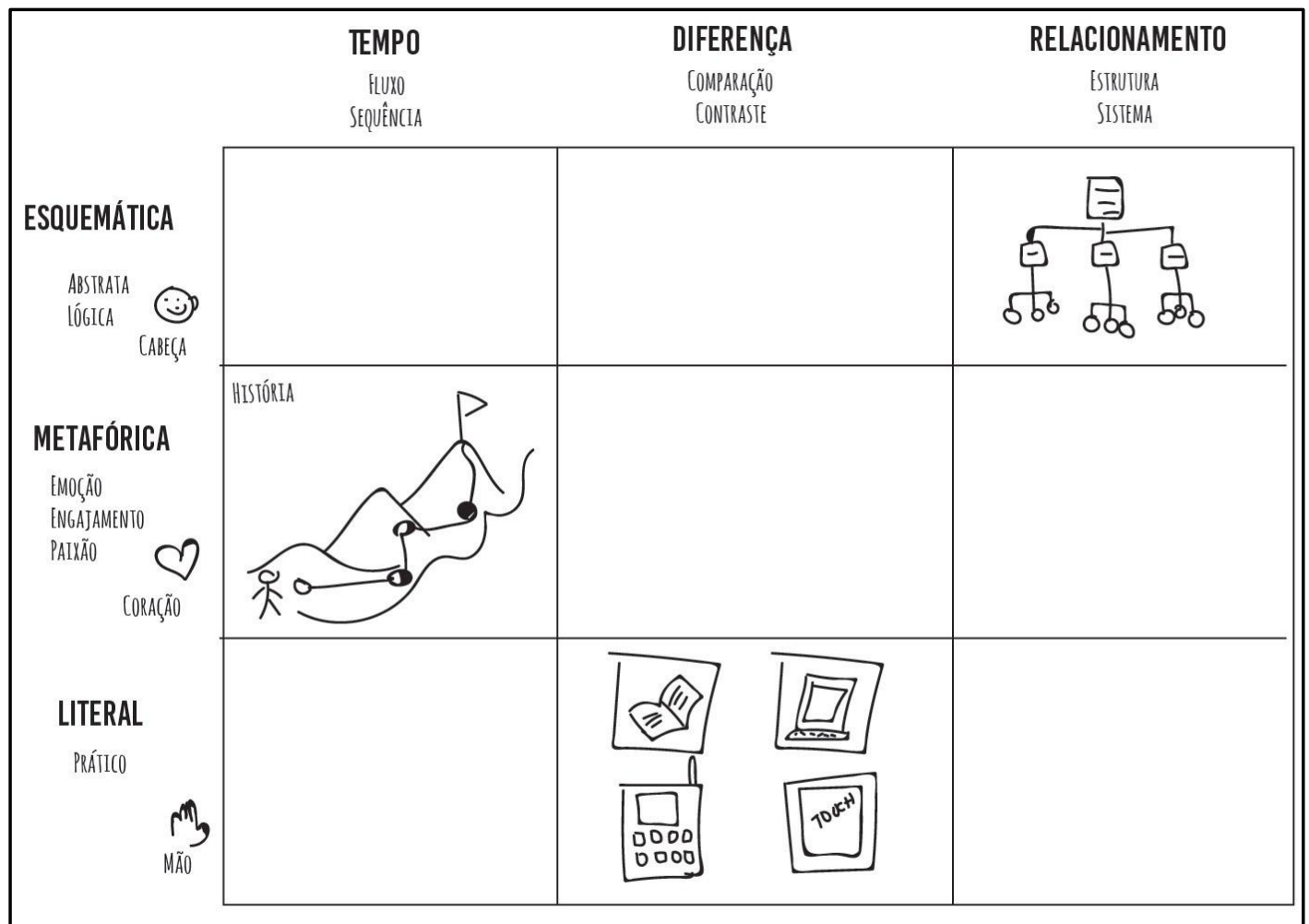

Figura 3: Representações para serem elaboradas na ferramenta Mural, baseada em Gray (2012).

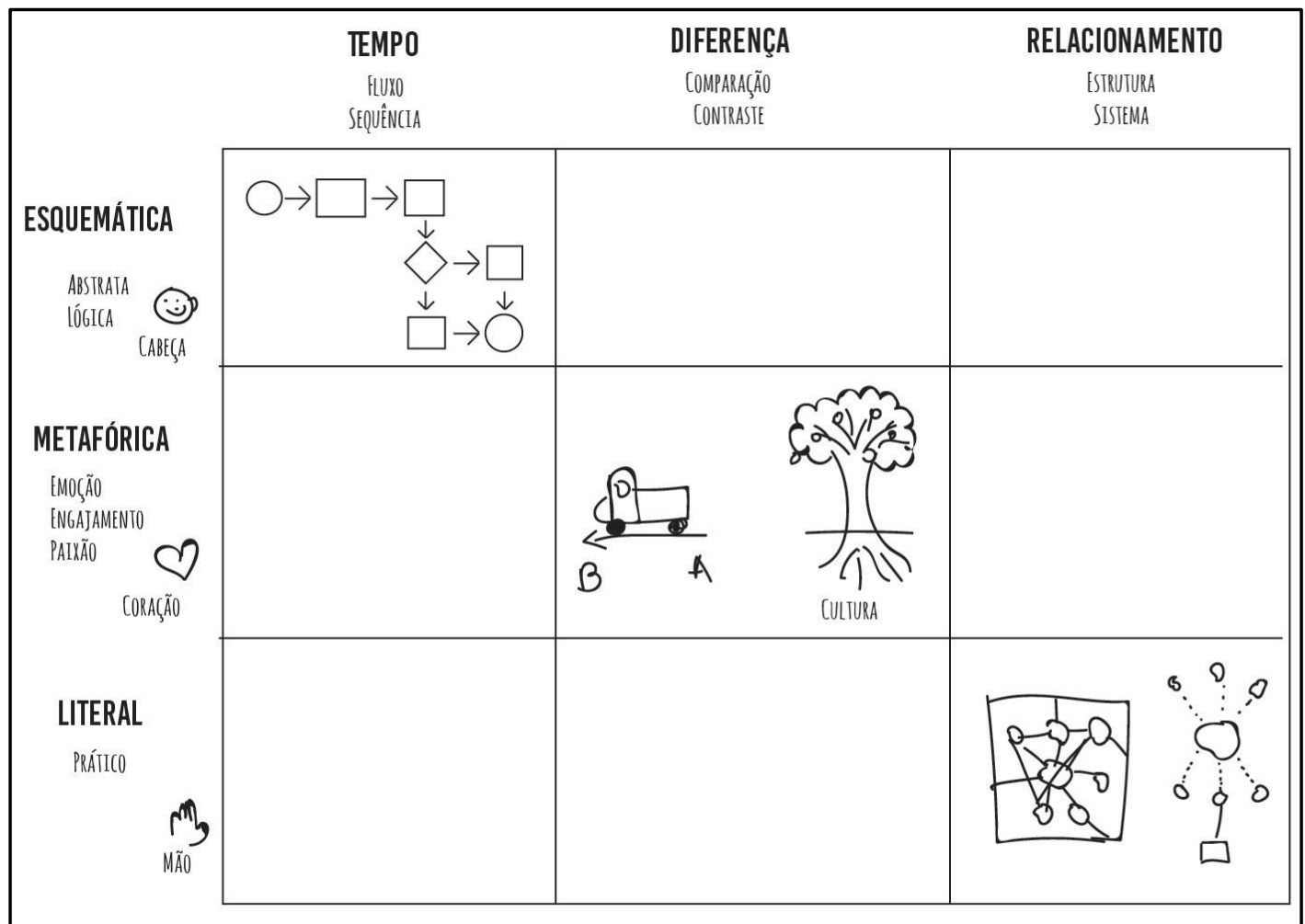


Figura 4: Representações para serem elaboradas na ferramenta Jamboard, baseada em Gray (2012).

\begin{tabular}{|c|c|c|c|}
\hline \multirow[b]{2}{*}{ 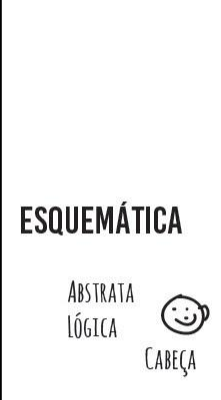 } & $\begin{array}{l}\text { TEMPO } \\
\text { Fluxo } \\
\text { SEQÛ̀NCIA }\end{array}$ & $\begin{array}{c}\text { DIFERENÇA } \\
\text { Compraraç̄o } \\
\text { Conrrasit }\end{array}$ & $\begin{array}{c}\text { RELACIONAMENTO } \\
\text { EsTRUUURA } \\
\text { SISTEMA }\end{array}$ \\
\hline & & $\prod_{E}^{(A) B}$ & \\
\hline 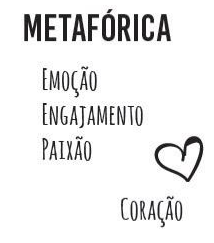 & & & Gravidade \\
\hline $\begin{array}{l}\text { LITERAL } \\
\text { Próitico } \\
\\
\\
\\
\text { Mão }\end{array}$ & 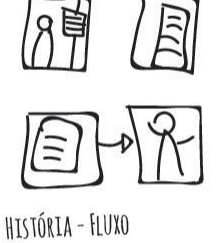 & & \\
\hline
\end{tabular}

\section{Análise dos dados e discussão dos resultados}

\section{Pré-diagnóstico baseado nas heurística de Nielsen}

O pesquisador realizou um pré-diagnóstico das ferramentas que foi revisado enquanto os participantes interagiam entre si e com as ferramentas Miro, Mural e Jamboard. Este prédiagnóstico apontou, quanto à visibilidade do estado do sistema, que as três ferramentas não permitem a visualização das configurações dos elementos manipulados que estão sendo alterados pelos outros usuários. Quanto à consistência e padrões/convenções, foi observado que os recursos de inserir e alterar as configurações dos elementos, embora não sejam difíceis de serem aprendidos, não seguem o padrão da maioria das ferramentas de edição conhecidas pelo pesquisador. As ferramentas Miro e Mural possuem recursos e formas de manipulação parecidos. A Jamboard é a mais discrepante neste sentido, pois os recursos de manipulação de elementos gráficos são mais simples e inferiores àqueles disponíveis na ferramenta Apresentação do pacote G-suíte ao qual a Jamboard está vinculada. Quanto à prevenção de erros e ao reconhecimento, correção e recuperação de erros, as ferramentas Miro e Mural apresentam problemas na manipulação colaborativa dos elementos: se dois usuários tentarem manipular as configurações de um mesmo elemento, o primeiro usuário fará as alterações e o segundo desencadeará a duplicação do elemento na página de trabalho. Este procedimento não emite nenhum alerta de erro e o usuário acredita ter executado a ação pretendida. $A$ Jamboard permite a alteração de diferentes configurações por usuários distintos trabalhando no mesmo elemento. Mesmo não sendo considerados problemas graves, são situações que 
podem impactar, em algum grau, a satisfação dos usuários. Estes problemas, identificados no pré-diagnóstico, foram confirmados na observação das dinâmicas de discussão visual que serão descritas neste trabalho.

\section{Interação e colaboração na ferramenta Miro}

Os participantes iniciaram a interação declarando que apesar de ter um conhecimento prévio, não tinham experiência "sólida" no uso da ferramenta Miro. Durante a realização da dinâmica, os participantes comentaram que a experiência anterior com a ferramenta Miro era bastante incipiente, haviam utilizado a ferramenta apenas uma vez para realização de uma tarefa sem complexidade. A partir deste relato, acredita-se que os resultados obtidos na dinâmica não foram influenciados por esta experiência anterior. Dialogando entre si, os participantes descobriram as principais funções necessárias para a realização colaborativa da tarefa proposta: (1) identificaram que não é possível trabalhar juntos no mesmo objeto (quando manipulados ao mesmo tempo, o objeto é duplicado e cada usuário trabalha no seu próprio objeto); (2) durante a manipulação dos objetos na área de desenho, só é possível visualizar a movimentação do cursor dos outros usuários. Entretanto, o resultado final da aplicação da configuração é visível a todos os usuários.

Ambos os participantes afirmaram que o período de cerca de 10 minutos é suficiente para aprender/descobrir as principais funções da ferramenta. Isso porque durante a interação ainda é possivel auxiliar o outro participante quando necessário. Neste processo de reconhecimento da ferramenta, os participantes mencionaram a falta do recurso de áudio ou videoconferência. $\mathrm{Na}$ aplicação dessa dinâmica foi usada a versão gratuita da ferramenta Miro. A versão completa da ferramenta Miro possui dentre outros recursos, chat e áudio e videoconferência. Um dos participantes sugeriu o uso dos post-its como forma de colocar recados aos demais participantes da tarefa. Para esse contexto colaborativo sem anonimato entre os participantes, a ferramenta também foi considerada adequada. Porém, considerando outros contextos onde o anonimato pode ser desejável no processo colaborativo, esta ferramenta não é adequada, pois os participantes são identificados por meio do login na ferramenta e os cursores de todos os participantes apresentam uma descrição identificando o usuário a quem pertence.

Quanto aos canais de comunicação, somente a versão completa da ferramenta apresenta chat, comentários, videoconferências, modo de apresentação, compartilhamento de tela, votação, timer e histórico $(\mathrm{log})$ de todas as ações executadas sobre os objetos. Porém não apresenta um diretório de armazenamento ou gerenciamento de arquivos definitivos ou provisórios. Esse recurso foi apontado como algo importante para a colaboração. O resultado final da elaboração das representações pode ser observado na Figura 5. 
Figura 5: Representações elaboradas na ferramenta Miro.

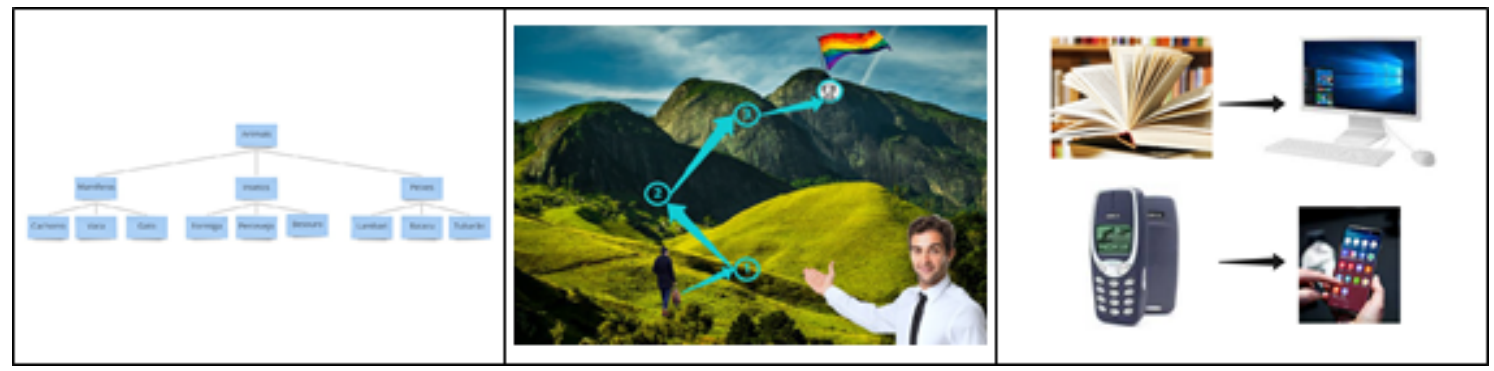

\section{Interação e colaboração na ferramenta Mural}

Os participantes declararam que não tinham experiência de uso ou conhecimento sobre a ferramenta Mural. Conversando durante a atividade, detectaram algumas características importantes para a realização colaborativa da tarefa proposta: (1) identificaram que não é possivel trabalhar juntos no mesmo objeto (quando manipulados ao mesmo tempo, o objeto assume a cor indicativa de cada usuário e se torna indisponível para seleção por outros usuários); (2) durante a manipulação dos objetos na área de desenho, só é possível visualizar a movimentação do cursor dos outros usuários sem saber que tipo de configuração está sendo realizada no objeto manipulado. Também perceberam que os recursos de chat, comentários e histórico $(\mathrm{log})$ de atividades, diretamente ligadas à colaboração, estão disponíveis a todos os usuários. O criador do arquivo é considerado o facilitador da reunião e a ele está associado o gerenciamento de outras funções como timer, votação e configuração do anonimato na colaboração.

Para esta ferramenta, os participantes concordaram que um período de 10 minutos é suficiente para aprender as principais funções básicas da ferramenta. Nesse reconhecimento da ferramenta, os participantes identificaram que o recurso de chamada de áudio e chat entre os participantes está disponível. Entretanto, não apresenta um diretório de armazenamento ou gerenciamento de arquivos definitivos ou provisórios. Ao acionar a função de download do trabalho realizado, um link para download do arquivo PDF ou PNG é enviado ao email do usuário. O resultado final da elaboração das representações pode ser observado na Figura 6.

Figura 6: Representações elaboradas na ferramenta Mural.

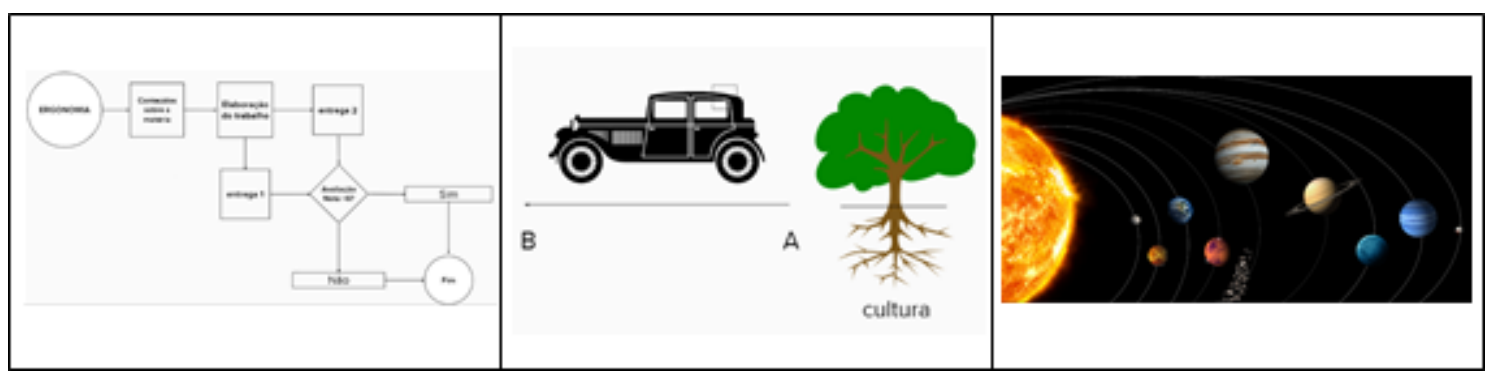




\section{Interação e colaboração na ferramenta Jamboard}

Os participantes declararam que não tinham experiência de uso ou conhecimento sobre a ferramenta Jamboard. No processo colaborativo, foram capazes de elaborar as três sugestões de representações. O resultado final da elaboração das representações pode ser observado na Figura 7.

Figura 7: Representações elaboradas na ferramenta Jamboard.

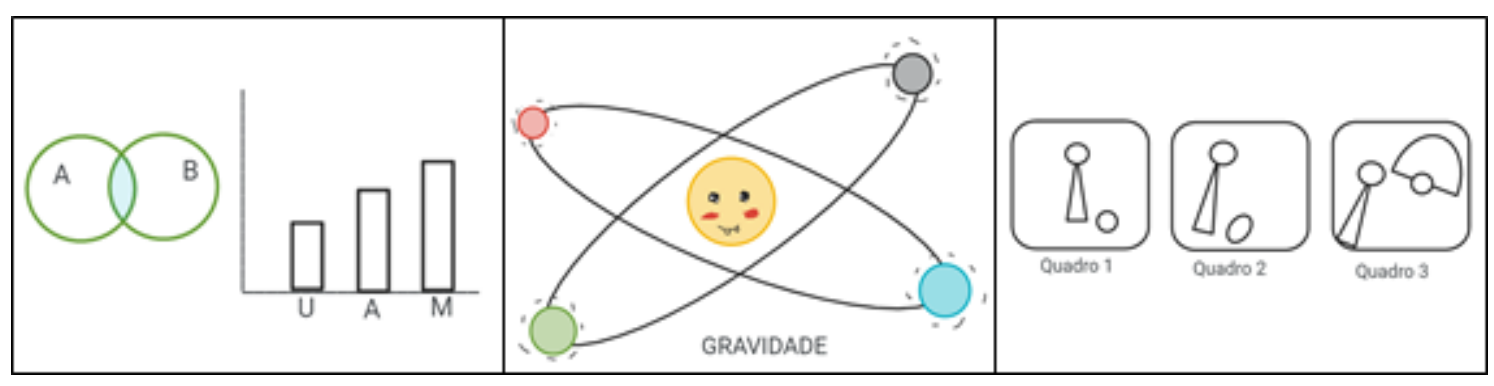

Dialogando entre si, os participantes descobriram as principais funções necessárias para a realização colaborativa da tarefa proposta: (1) identificaram que é possível trabalhar juntos no mesmo objeto (em configurações diferentes); (2) durante a manipulação dos objetos na área de desenho, só é possível visualizar a movimentação do cursor dos outros usuários, sem saber que tipo de configuração está sendo realizada no objeto manipulado; entretanto, o resultado final da aplicação da configuração é visível a todos os usuários. Também perceberam a ausência de recursos como chat e comentários para comunicação entre os participantes.

O período de 10 minutos foi considerado suficiente para descobrir as principais funções da ferramenta. Neste processo de reconhecimento da ferramenta, considerando a interface e os comandos disponíveis, a ferramenta foi considerada adequada ao nível de conhecimento dos participantes, pois é de fácil aprendizado e de fácil utilização. Porém, afirmam que os recursos são limitados e limitantes, impedindo a produção de representações mais bem elaboradas. A ferramenta não apresenta consistência em relação a outros sistemas usados anteriormente (Google Presentation que faz parte do mesmo pacote de sistemas possui mais recursos de formas básicas e configurações). A ferramenta também não permite a colaboração anônima; todos os participantes são identificados e a foto do perfil do usuário aparece para os demais participantes na posição do cursor. Quanto a canais de comunicação, a ferramenta também não apresenta canais adequados para a colaboração, tais como chamada de áudio/vídeo, compartilhamento de tela, votação, timer e histórico $(\log )$ das ações executadas sobre os objetos. Foi especulado entre os participantes que talvez a ausência destes recursos se deva ao fato de a ferramenta Jamboard pertencer ao $G$ Suite, composto por outras ferramentas que podem satisfazer essas necessidades de comunicação (Drive para armazenamento de arquivos, Meet para reuniões e videochamadas, Presentation para slides com imagens e formas básicas etc). 


\section{Funções e recursos que auxiliam uma discussão visual}

A partir da realização de dinâmicas colaborativas com as três ferramentas, os participantes identificaram recursos que são considerados importantes para a realização de uma dinâmica colaborativa para discussão visual. Esses recursos foram organizados em três grupos conforme sua relação com a comunicação entre os participantes, com a manipulação dos elementos na representação e com a organização do trabalho. Esta lista de recursos pode ser observada na tabela 2 .

Tabela 2: Funções e recursos que auxiliam na discussão visual

\begin{tabular}{|c|c|}
\hline Grupos & Funções e recursos \\
\hline $\begin{array}{l}\text { Comunicação } \\
\text { entre os usuários }\end{array}$ & $\begin{array}{l}\text { - Disponibilizar um chat entre os usuários; } \\
\text { - Realizar chamada de áudio; } \\
\text { - Enviar mensagem de áudio; } \\
\text { - Realizar chamada de vídeo }\end{array}$ \\
\hline $\begin{array}{l}\text { Manipulação dos } \\
\text { elementos na } \\
\text { representação }\end{array}$ & $\begin{array}{l}\text { - Enviar mensagens de erro/alerta quando um participante for usar um objeto } \\
\text { que já está sendo manipulado; } \\
\text { - Permitir que o mesmo objeto seja usado por mais de um participante quando } \\
\text { configurações diferentes forem alteradas (por exemplo, uma caixa de texto } \\
\text { pode ter o rótulo alterado por um participante enquanto o outro altera a cor } \\
\text { da borda ou o preenchimento); } \\
\text { - Possibilitar a marcação de um objeto como restrito a um usuário; } \\
\text { - Permitir a visualização da caixa de ferramentas aplicável ao objeto que está } \\
\text { sendo manipulado pelos outros usuários; }\end{array}$ \\
\hline $\begin{array}{l}\text { Organização do } \\
\text { trabalho }\end{array}$ & $\begin{array}{l}\text { - Disponibilizar um diretório de armazenamento ou gerenciamento de arquivos } \\
\text { - } \text { Pefinitivos ou provisórios; } \\
\text { - Permitir o registro de comentários sobre os elementos da representação; } \\
\text { - Permitir o compartilhamento de tela (não apenas do arquivo) entre os } \\
\text { - } \text { usuários; } \\
\text { - Prganizar uma votação; } \\
\text { - Regsibilitar a definição de um tempo para a execução da tarefa (timer); } \\
\text { - Fazer download do arquico de ações executadas sobre os objetos; } \\
\text { - Banco de imagens e ícones para facilitar o uso de imagens nas } \\
\text { - } \text { Enviar email aos usuários no caso de alteração do arquivo após a sessão de } \\
\text { - colaboração síncrona. }\end{array}$ \\
\hline
\end{tabular}

\section{Considerações finais}

Este artigo investigou as ferramentas colaborativas online para facilitação visual Jamboard, Miro e Mural, a partir da realização de um pré-diagnóstico (com base nas heurísticas de usabilidade de Nielsen, 1990), seguido de uma pesquisa com usuários interagindo diretamente com as ferramentas. Todas as ferramentas apresentaram apenas problemas de baixa 
severidade. Entretanto, o maior diferencial entre elas se refere aos recursos disponíveis para elaborar as representações e às funções e recursos comunicacionais que possibilitem a realização de dinâmicas colaborativas para discussão visual.

A partir dos procedimentos adotados durante a pesquisa e da exposição dos resultados, considera-se que é possível realizar dinâmicas colaborativas para discussão visual com as três ferramentas avaliadas, embora os resultados das representações tenham sido melhor elaborados nas ferramentas Miro e Mural do que na Jamboard.

Para trabalhos futuros, sugere-se estudar a realização de dinâmicas com uma quantidade maior de participantes em cada sessão, a fim de verificar se essas ferramentas apresentam desempenho satisfatório na realização de atividades colaborativas com mais de dois usuários.

\section{Agradecimento}

À Universidade Federal do Maranhão e ao Programa de Pós-Graduação em Design.

À Universidade Federal do Paraná e ao Programa de Pós-Graduação em Design. O presente trabalho foi realizado com apoio da Coordenação de Aperfeiçoamento de Pessoal de Nível Superior - Brasil (CAPES) - Código de Financiamento 001.

\section{Referências}

Arnheim, R. (1969). Visual thinking. Berkeley: University of California Press.

Bertolini, E. A. S., \& Silva, M. A. M. (2005). Metacognição e motivação na aprendizagem: relações e implicações educacionais. Revista Técnica IPEP, 5(1/2), 51-62.

Cyrs, T. E. (1997). Visual thinking: let them see what you are saying. New directions for teaching and learning, 71, 27-32.

Engelhardt, Y. (2002). The Language of Graphics: a framework for the analysis of syntax and meaning in maps, charts and diagrams. Amsterdam: ILLC- Publications.

GOOGLE. (n.d.). What's Jamboard? Disponível em: <https://support.google.com/jamboard/ans wer/7424836 ?hl=en>. Acesso em: 31/03/2021.

Gray, D. (2012). Gamestorming: jogos corporativos para mudar, inovar e quebrar regras. Rio de Janeiro: Alta Books.

Hardy, K., \& Garfen, D. (2020). Driving Innovation and Collaboration Remotely. ITNOW, 62(3), 21-23.

Howe J., Parker S. H., Wiggermann N., \& Zagarese V. (2021). The Role of Health Care Human Factors in Responding to COVID-19: Findings From an Industry Survey. Ergonomics in Design. 29(1) 29-32.

ITU-T. (2006). H.323 - Packet-based multimedia communications systems. International Telecommunication Union. [Online]. Disponível em <http://www.itu.int/rec/T-REC-H.323/e>.

ITU-T. (2007). T.120 - Data protocols for multimedia conferencing. International Telecommunication Union. [Online]. Disponível em <http://www.itu.int/rec/T-REC-T.120/en>. 
LUCIDSPARK. (2020). Report: How collaboration and creativity are suffering in the wake of COVID-19. Disponível em: <https://lucidspark.com/blog/report-collaboration-and-creativityduring-covid>. Acesso em: 31/03/2021.

MIRO. (n.d.). An online visual collaboration platform for teamwork. Disponível em: $<$ https://www.miro.com>. Acesso em: 31/03/2021.

MURAL. (n.d.). More than a whiteboard. Disponível em: <https://www.mural.co>. Acesso em: $31 / 03 / 2021$.

Nielsen, J., Molich, R. (1990). Heuristic evaluation of user interfaces. Proc. ACM CHI'90 Conf., Seattle, EUA, 1-5 abril, p. 249-256.

Novak, J. D., \& Cañas, A. J. (2006). The theory underlying concept maps and how to construct them. Florida Institute for Human and Machine Cognition, 1.

Roam. D. (2009). The Back of the Napkin: Solving Problems and Selling Ideas with Pictures. Penguin Group, New York.

Roush, C., \& Burmeister, A. R. (2020). COVID-19 and the Central Dogma: an Activity To Improve Student Learning and Engagement. Journal of microbiology \& biology education, 21(3), 21.3.68.

Sibbet, D. (2006). Graphic facilitation: transforming group process with the power of visual listening. San Francisco: The Grove Consultants International.

Singh, G., Hawkins, L., \& Whymark, G. (2009). Collaborative knowledge building process: an activity theory analysis. VINE: The journal of information and knowledge management systems, 39(3), 223-241.

Stahl, G. (2006). Group cognition: computer-support for building collaborative knowledge. Cambridge: The MIT Press.

\section{Sobre os autores}

Reinaldo Pereira de Moraes, Me, UFPR, Brasil <reinaldomoraes@ufpr.br> Claudia Mont'Alvão, Dra., UFMA, Brasil <cmontalvao@puc-rio.br> Stephania Padovani, Dra., UFPR, Brasil <stephania.padovani@gmail.com> 